\title{
BALANCE POINT AND STABILITY OF VECTOR BUNDLES OVER A PROJECTIVE MANIFOLD
}

\author{
XiaOWEI WANG
}

\begin{abstract}
In this paper, we show that stability of a vector bundle over a projective manifold is equivalent to the embedding of the base manifold induced by the global sections into the Grassmanian can be moved to a balanced place in the sense of Donaldson, thus verifying a conjecture of Donaldson.
\end{abstract}

\section{Introduction}

The stability of a vector bundle over a projective manifold has been studied extensively. In order to construct a good moduli space for the bundles over a projective variety, various notions of stability have been introduced. Mumford first introduced Mumford stable vector bundles (c.f. [NS]). Later Gieseker introduced a new notion of stability which is now called Gieseker stablility when he was constructing the moduli space of vector bundles over complex surfaces. It turns out that the notion of Gieseker stablility of vector bundles fits nicely into Mumford's Geometric Invariant Theory, so the GIT machinery of constructing moduli spaces can be effectively applied to this case. (c.f. [S])

The differential-geometic picture of stability turns out to be very fruitful as well. The Mumford stability of a vector bundle fits perfectly into the infinite dimensional symplectic quotient picture. Specifically, Mumford stability of a vector bundle over a projective manifold is equivalent to the existence of HermitianYang-Mills metric, which amounts to solving a 2nd-order elliptic PDE arising from the moment map of a Hamiltonian action of the infinite dimensional gauge group on the affine space of connections on that vector bundle. This correspondence was established by Donaldson and Uhlenbeck-Yau (c.f. [D2],[D3],[UY]).

But for Gieseker stability, the differential-geometric interpretation of this condition remained somewhat unclear until Leung's thesis [Le] first gave a differential-geometric and also a symplectic quotient interpretation. Recently, in [D4], inspired by the work of Luo (c.f. [Luo]) Donaldson suggested an analogous way of looking at the stability problem for vector bundle. Instead of looking at the space of connections, he looked at the mapping space of the base manifold into the Grassmannian. He conjectured a geometric criterion which is very similar to Luo's criterion for stability of projective manifold, namely the Balanced place

Received September 26, 2001.

Revised version received March 3, 2002. 
condition (see the following theorem) of the embedding is equivalent to the stability of a holomorphic vector bundle over a Riemann surface. This point of view was also taken by Uhlenbeck and Yau [UY], in their paper, in order to prove the existence of Hermitian-Yang-Mills metric, they perturb the equation and study the limit of the perturbed equation as the perturbation parameter goes to zero. In that case if the vector bundle is not Mumford stable then the solution will blow up and this blowing up give rise to an meromorphic map from the base manifold into Grassmanian which pulls back a destablizing subsheaf of the original vector bundle. The author also learned from Professor Yau that he and Conan Leung have had the similar idea to attack the stability for vector bundles over almost complex manifolds.

Our main result of this paper is to confirm Donaldson's conjecture. Actually what we show is a little more general. We obtain the same conclusion for vector bundles over any projective manifold, and the correct notion of stability should be in the sense of Gieseker whereas in the Riemann surface case Gieseker stability and Mumford stability are the same.

Theorem 1.1. Let $E$ be a holomorphic vector bundle over a polarized projective manifold $\left(X, \mathcal{O}_{X}(1)\right), E(k):=E \otimes \mathcal{O}_{X}(k)$. Then $E$ is Gieseker polystable if and only if there is a number $M$ such that for $k>M$, the embedding:

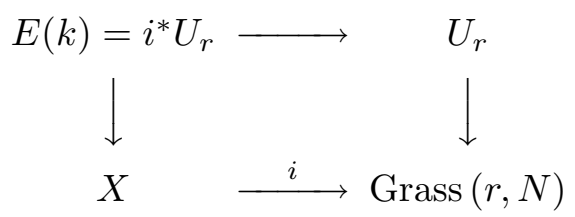

given by the global sections of $H^{0}(X, E(k))$ can be moved to a balanced point, i.e. there is a $g \in S L(N)$ which is unique up to left translation by $S U(N)$, where $N=\operatorname{dim} H^{0}(X, E(k))$ such that:

$$
\frac{1}{\operatorname{Vol}(X)} \int_{g \cdot X} A(x) A(x)^{*} d \operatorname{Vol}_{X}=\frac{r}{N} I_{N \times N}
$$

where $A:=A_{N \times r}=\left[\overrightarrow{a_{1}}, \cdots, \overrightarrow{a_{r}}\right]$ and $\overrightarrow{a_{1}}, \cdots, \overrightarrow{a_{r}} \in \mathbb{C}^{N}$ is an orthonormal basis of the corresponding r-plane in $\operatorname{Grass}(r, N)$, i.e. $A$ is the coordinate for $\operatorname{Grass}(r, N) . I_{N \times N}$ is the identity matrix.

The paper is organized as follows. In Section 2, we recall some basic facts of GIT, introduce various notions of stability, and finally give some equivalent criteria like how to tell if a vector bundle over a projective manifold is Gieseker stable or not. In Section 3, we give a symplectic picture of the mapping space and study the relation between the position of the base manifold in the Grassmanian and the stability of the vector bundle. The main theorem will be proven in this section. In Section 4, we describe the differential geometric interpretation of the Balanced point in the mapping space and its relation to Leung's equation and the heat kernel. 


\section{Gieseker Stability of Vector Bundles}

In this section we are going to give the GIT criteria for Gieseker stability of a vector bundle over a polarized projective manifold.

Definition 2.1. Let $\left(X, \mathcal{O}_{X}(1)\right)$ be a polarized projective manifold with a very ample line bundle $\mathcal{O}_{X}(1)$, and $E$ be a vector bundle over $X$. Define

$$
\chi(E):=\Sigma(-1)^{i} h^{i}(X, E)
$$

where $h^{i}(X, E)=\operatorname{dim} H^{i}(X, E)$. We call it Gieseker stable (resp. semi-stable) if for any subsheaf $F \subset E$,

$$
\frac{\chi(E(k))}{r k(E)}>(r e s p . \geq) \frac{\chi(F(k))}{r k(F)}
$$

for $k>>1$, where $E(k):=E \otimes \mathcal{O}_{X}(k), F(k):=F \otimes \mathcal{O}_{X}(k)$.

$A$ Gieseker semistable vector bundle is called Gieseker polystable if $E$ is a direct sum of Gieseker stable vector bundles.

\section{Remark 2.1.}

(i) It is easy to see $E$ is Gieseker stable (resp. semi-stable) if and only if $E(m)$ is for some $m$.

(ii) This numerical criterion actually comes from the numerical criterion due to Mumford and Hilbert in GIT.

Now pick a number $k>>1$ such that $E(k)$ is globally generated.

Definition 2.2. Define Quot $(\mathcal{H}, p):=\{$ Quot-Scheme of $\mathcal{H}$ with Hilbert polynomial $p\}$, where $\mathcal{H}:=V \otimes \mathcal{O}_{X}(-k)$, and $V:=H^{0}(X, E(k))$

Now E can be viewed as a point

$$
[\rho: \mathcal{H} \rightarrow E \rightarrow 0] \in \operatorname{Quot}(\mathcal{H}, p)
$$

$S L(V)$ acts natuarlly on $\operatorname{Quot}(\mathcal{H}, p)$ as follows

$$
[\rho] \circ g:=[\rho \circ g]
$$

where $g \in S L(V)$. Now let us recall some basic facts in GIT :

Definition 2.3. (compare with Definition 3.4) Let $X$ be a projective scheme. $A$ point $x \in X$ is semistable with respect to a G-linearized ample line bundle $L$ if there is an integer $n$ and an invariant global section $s \in H^{0}\left(X, L^{\otimes k}\right)$ with $s(x) \neq 0$. We say $x$ is stable if in addition, the stabilizer $G_{x}$ is finite and the $G$-orbit of $x$ is closed in the open set of all semistable points in $X$.

In order to check the stability of a point we have the following useful criterion. Let $\lambda: \mathbb{G}_{m} \mapsto G$ be an non-trivial one-parameter subgroup of $G$. Then the action of $G$ on $X$ induces an action of $\mathbb{G}_{m}$ on $X$. Since $X$ is projective, the orbit map $\mathbb{G}_{m} \rightarrow X, t \mapsto \lambda(t) \cdot x$ extends uniquely to a morphism $f: \mathbb{A}^{1} \rightarrow X$ such that the diagram 


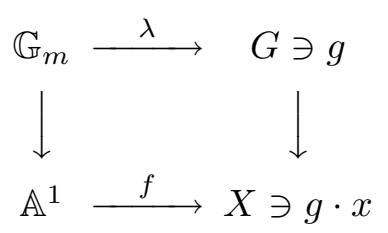

commutes, where $\mathbb{G}_{m}=\mathbb{A}^{1}-\{0\} \rightarrow \mathbb{A}^{1}$ is the inclusion. We write

$$
\lim _{t \rightarrow 0} \lambda(t) \cdot x:=f(0)
$$

Clearly, $f(0)$ is a fixed point of the action of $\mathbb{G}_{m}$ on $X$ via $\lambda$. In particular, $\mathbb{G}_{m}$ acts on the fiber $L_{f(0)}$ with weight $r$. Define the number $\mu^{L}(x, \lambda):=-r$. The following lemma is well-known (c.f. $[\mathrm{MFK}]$ )

Lemma 2.1. (Hilbert-Mumford Criterion) A point $x \in X$ is semistable if and only if for all non-trivial one-parameter subgroups $\lambda: \mathbb{G}_{m} \rightarrow G$ one has

$$
\mu^{L}(x, \lambda) \geq 0
$$

$x$ is stable if and only if strict inequality holds for all non-trivial $\lambda$.

In particular, if $X$ is a projective space $\mathbb{P} V$ and $L$ is the hyperplane bundle $\mathcal{O}_{X}(1)$ and the action by a reductive group $G^{\mathbb{C}}$ is via a representation $G^{\mathbb{C}} \rightarrow$ $S L(V)$, then we can talk about the stable points in the GIT sense.

Definition 2.4. Suppose $v \in \mathbb{P} V$ and let $\bar{v} \in V$ denote its lift in $V$. Then $v$ is called:

a) semistable if the closure of the $G^{\mathbb{C}}$-orbit $\overline{G^{\mathbb{C}} \cdot \bar{v}}$ does not contain 0 ,

b) stable if $G^{\mathbb{C}} \cdot \bar{v}$ is closed and the stablizer $G_{v}^{\mathbb{C}}$ is finite.

Kempf and Ness $[\mathrm{KN}]$ proved the following theorem:

Theorem 2.1. Let $G^{\mathbb{C}}$ be a reductive algebraic group with maximal compact group $G$, and let $V$ be a vector space with a $G$-invariant Hermitian metric $h$. $G^{\mathbb{C}}$ acts on $V$, so it acts on $\mathbb{P} V$, and $\mathcal{O}(1)$ is the linearized bundle over it. Then a point $v \in \mathbb{P} V$ is stable if and only if the function $S_{v}(g):=\|g \cdot \bar{v}\|_{h}^{2}: G^{\mathbb{C}} / G \rightarrow \mathbb{R}$ is proper, where $\bar{v} \in V$ is a lift of $v \in \mathbb{P} V$.

Now we come back to our discussion of vector bundles over $X$. Let $R$ be the open set of semi-stable sheaves in $\operatorname{Quot}(\mathcal{H}, p), \mathcal{E}$ the universal quotient sheaf over $\operatorname{Quot}(\mathcal{H}, p)$. Then we have the following diagram:

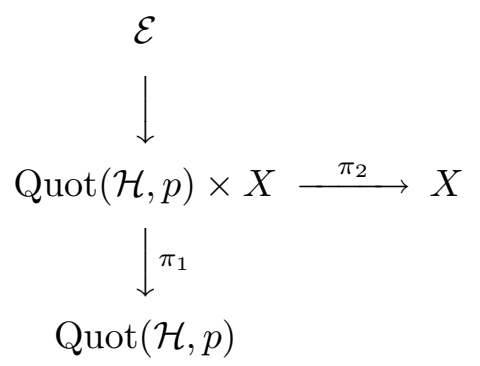


Let $L_{k}:=\operatorname{Det}\left(\left(\pi_{1}\right)_{*}\left(\mathcal{E} \otimes\left(\pi_{2}\right)^{*} \mathcal{O}_{X}(k)\right)\right)$ be the determinant line bundle over Quot $(\mathcal{H}, p)$. It is very ample and linearized with respect to the $S L(V)$ action on $\operatorname{Quot}(\mathcal{H}, p)$ in the GIT sense for $k>>1$. Thus we can speak of semistable and stable points in the closure $\bar{R}$ of $R$ in $\operatorname{Quot}(\mathcal{H}, p)$ with respect to $L_{k}$ and the $S L(V)$ action. The following theorem is due to Simpson [S], which justifies the equivalence between the Gieseker slope stability in Definition 2.1 and GIT stability.

Theorem 2.2. Suppose that $m$ and, for fixed $m$, also $k$, are sufficiently large integers. Then $R=\bar{R}^{s s}\left(L_{k}\right)$ and $R^{s}=\bar{R}^{s}\left(L_{k}\right)$, where $\bar{R}^{s s}\left(L_{k}\right)$ (resp. $\bar{R}^{s}\left(L_{k}\right)$ ) are the semistable (resp. stable) points of $\bar{R}$ with respect to the linearization $L_{k}$. Moreover, the orbit of a point $[\rho: \mathcal{H} \rightarrow F]$ is closed in $R$ if and only if $F$ is Gieseker polystable.

Let $\mathcal{M}(X, p(k))$ denote the moduli space of Gieseker stable sheaves with Hilbert polynomial $p(k)$, then an immediate corollary is the following (c.f. $[\mathrm{S}]$ ).

Corollary 2.1. $\mathcal{M}(X, p(k))$ is a quasi-projective scheme and it is the GIT quotient $\bar{R}^{S}\left(L_{k}\right) / / S L(V)$.

In order to prove the main theorem of this paper, we need to use another stability criterion proved by Gieseker[G].

Definition 2.5. Suppose $E$ is a rank $r$ globally generated vector bundle on $X$, and $\left\{s_{1}, \ldots, s_{N}\right\} \in H^{0}(E)$ a basis. Let $T(E) \in \operatorname{Hom}\left(\wedge^{r} H^{0}(E), H^{0}(\operatorname{det}(E))\right)$ be defined by

$$
T(E)\left(s_{i_{1}}, \ldots, s_{i_{r}}\right):=s_{i_{1}} \wedge \ldots \wedge s_{i_{r}} \in H^{0}(\operatorname{det} E)
$$

we can view $T(E)$ as a point in $\mathbb{P H o m}\left(\wedge^{r} H^{0}(E), H^{0}(\operatorname{det}(E))\right) ; S L\left(H^{0}(E)\right)$ acts on it and the linearized bundle is $\mathcal{O}(1)$ over $\mathbb{P H o m}\left(\wedge^{r} H^{0}(E), H^{0}(\operatorname{det}(E))\right)$. Again we can sat whether the point

$$
T(E) \in \mathbb{P} \operatorname{det}\left(\wedge^{r} H^{0}(E), H^{0}(\operatorname{det}(E))\right)
$$

is stable or not. In particular we have the following theorem due to Gieseker (c.f. $[\mathrm{G}],[\mathrm{HL}]$ ).

Theorem 2.3. There is an $M$ so that if $k>M$, then $E(k)$ is Gieseker stable (resp. semistable) if and only if $T(E(k))$ is stable (resp. semistable) under the action of $S L\left(H^{0}(E(k))\right.$. Moreover, the orbit of $T(E(k))$ is closed if and only if $E(k)$ is Gieseker polystable.

Remark 2.2. Actually, Gieseker proved a bit more, in order for the theorem above to hold we only need to assume that $E$ is a torsion free sheaf.

\section{Symplectic Quotient Picture}

In this section, let us first recall some basic facts from geometric quantization and the symplectic quotient picture which can be found in e.g.[DK]. Let $(V, \omega)$ be a Kähler manifold. 
Definition 3.1. We say that $(V, L)$ is quantized if $(L, h)$ is a holomorphic Hermitian line bundle $\pi: L \rightarrow V$ with Ric $(h)=\omega$.

To be more explicit, the Hermitian structure defines a function $h: L \rightarrow \mathbb{R}^{+}$ by $h\left(\xi_{p}\right)=\left\|\xi_{p}\right\|_{p}^{2}$ where $\xi_{p} \in \pi^{-1}(p)$ and $\|\cdot\|_{p}$ is the Hermitian norm. Then let

$$
\alpha=\frac{1}{4 \pi i}(\partial-\bar{\partial})(\log (h))=d^{c} \log (h) ;
$$

$\alpha$ is a 1 -form on $L-\{0\}$ and defines the canonical $S^{1}$ connection: if $\tilde{\xi}$ is the vector field generating the inifinitesimal action of $S^{1}$ on $\mathrm{L}$ given by multiplication by $e^{2 \pi i v}$ fiberwise, then $i_{\tilde{v}} \alpha=1$ and $\mathcal{L}_{\tilde{v}} \alpha=0$. We see that:

$$
\pi^{*}(\omega)=d \alpha=\frac{1}{2 \pi i} \partial \bar{\partial} \log h .
$$

Let $G$ be a compact Lie group, and let $G^{\mathbb{C}}$ be its complexification. We identify $\mathfrak{g}^{\mathbb{C}}=\mathfrak{g} \oplus i \mathfrak{g}$ where $\mathfrak{g}^{\mathbb{C}}$ and $\mathfrak{g}$ are the Lie algebras of $G^{\mathbb{C}}$ and $G$ respectively. We consider actions of $G^{\mathbb{C}}$ of the following type:

Assumption 3.1. The action of $G^{\mathbb{C}}$ on $V$ satisfies:

1) $G^{\mathbb{C}} \times V \longrightarrow V$ is holomorphic.

2) The induced action $G \times V \longrightarrow V$ acts by isometries with respect to the Kähler metric;

3) $G$ acts symplectically with respect to $\omega$.

Notice that 1) and 2) are equivalent to 1) and 3). Throughout the rest of this section we assume that $V$ is a quantized Kähler manifold and with a $G^{\mathbb{C}}$-action satisfying Assumption 3.1.

Definition 3.2. A linearization of the $G^{\mathbb{C}}$ action on $V$ is a holomorphic action of $G^{\mathbb{C}}$ on $L$ covering the action on $V$, and such that $G$ acts unitarily on the fibers.

We will use the notation that if $a \in \mathfrak{g}$ then $\tilde{a} \in \operatorname{Vect}(V)$ is the vector field generated by the infinitesimal action of $a$ and $\tilde{\tilde{a}} \in \operatorname{Vect}(L)$ is generated by its lift. Notice that $a \mapsto \tilde{a}$ is a anti-homomorphism of Lie algebras so that $\widetilde{[a, b}]=-[\tilde{a}, \tilde{b}]$. For any symplectic manifold $(V, \omega)$ with a symplectic action of a compact group $G$, we have the notion of a moment map.

Definition 3.3. An $\mathrm{Ad}^{*}$-equivariant moment map is a map satisfying the following: $\mu: V \longrightarrow \mathfrak{g}^{*}$ such that $d\langle\mu(x), a\rangle=i_{\tilde{a}} \omega(x)$ and $\langle\mu(g \cdot x), a\rangle=$ $\left\langle\mu(x), \operatorname{Ad}_{g}(a)\right\rangle$, where $a \in \mathfrak{g}$. In the following we will use the notation: $\mu_{a}(x):=$ $\langle\mu(x), a\rangle$.

Then we have the following proposition. (c.f. $[\mathrm{DK}],[\mathrm{B}]$ )

Proposition 3.1. A choice of a linearization uniquely determines a moment map and, conversely, a choice of a moment map uniquely determines a linearization. 
Definition 3.4. We say a point $x \in V$ is analytically stable for a linearized action of $G^{\mathbb{C}}$ on $V$ if for every non-zero $\xi_{x} \in L_{x}$ the function $g \rightarrow h\left(g \cdot \xi_{x}\right)$ is proper.

Remark 3.1. Notice that this definition is equivalent to Definition 2.4 in the case that $V=\mathbb{C P}^{N}$.

The following two lemmas are standard (c.f. [B]); for completeness, we include a proof here.

Lemma 3.1. (Analytic version of Hilbert-Mumford criterions) A point $x \in V$ is analytically stable with respect to the action of $G^{\mathbb{C}}$ if and only if it is analytically stable with respect to the restricted action of every real 1-parameter subgroup $t \rightarrow e^{i t a} \in G^{\mathbb{C}}$ where ita $\in i \mathfrak{g} \subset \mathfrak{g}^{\mathbb{C}}$.

Remark 3.2. This lemma is just the analytic version of Lemma 2.1.

Lemma 3.2. An orbit $G^{\mathbb{C}} \cdot x \subset V$ is analytically stable if and only if $G^{\mathbb{C}} \cdot x$ has no infinitesimal stablizers and $\mu=0$ somewhere on $G^{\mathbb{C}} \cdot x$. Furthermore, the set $G^{\mathbb{C}} \cdot x \cap \mu^{-1}(0)$ consists of exactly one $G$ orbit.

Proofs of Lemmas 3.1 and 3.2. Since the function $g \rightarrow h\left(g \cdot \xi_{x}\right)$ is constant along $G$ orbits, it descends to a function on $G^{\mathbb{C}} / G \cong \mathfrak{g}$ and is given by $a \rightarrow h\left(e^{i a} \cdot \xi_{x}\right)$. Consider the function $S_{x}: \mathfrak{g} \rightarrow \mathbb{R}$ defined by

$$
S_{x}(a)=\frac{h\left(e^{i a} \cdot \xi_{x}\right)}{h\left(\xi_{x}\right)}
$$

$S_{x}$ does not depend on the choice of $\xi_{x}$ and is proper if and only if $x$ is analytically stable. Lemma 3.1 is equivalent to the statement that $S_{x}$ is proper if and only if it is proper on each line through the origin.

For Lemma 3.2, assume first that $S_{x}$ is proper. Then for any $a \in \mathfrak{g}$, the real function defined by $L_{a}(t)=\log \left(S_{x}(t a)\right)$ is proper. We calculate that

$$
\begin{aligned}
\frac{d L_{a}(t)}{d t} & =\frac{\mathcal{L}_{J} \tilde{\tilde{a}}\left(L_{e^{i t a}}^{*} h\right)}{L_{e^{i t a}}^{*} h} \\
& =\left(\frac{\mathcal{L}_{J} \tilde{\tilde{a}}^{h}}{h}\right)\left(e^{i t a} \cdot \xi_{x}\right)
\end{aligned}
$$

where $L_{e^{i t a}}$ is the left multiplication by $e^{i t a}$. J is the complex structure, $J(\partial h)=$ $i \partial h$ and $J(\bar{\partial} h)=-i \bar{\partial} h$ so we have

$$
\begin{aligned}
\mathcal{L}_{J} \tilde{\tilde{a}} & =i_{J \tilde{\tilde{a}}} d h \\
& =i_{\tilde{\tilde{a}}} J(\partial h+\bar{\partial} h) \\
& =i_{\tilde{\tilde{a}}}(-4 \pi h \alpha) \\
& =4 \pi h \pi^{*}\left(\mu_{a}\right)
\end{aligned}
$$

and so

$$
\frac{d L_{a}(t)}{d t}=4 \pi \mu_{a}\left(e^{i t a} \cdot x\right)
$$


Similarly,

$$
\begin{aligned}
\frac{d^{2} L_{a}(t)}{d t^{2}} & =\left.\left(4 \pi \mathcal{L}_{J \tilde{a}} \mu_{a}\right)\right|_{e^{i t a} \cdot x} \\
& =\left.\left(4 \pi i_{J \tilde{a}} i_{\tilde{a}} \omega\right)\right|_{e^{i t a} \cdot x} \\
& =\left.\left(4 \pi\|\tilde{a}\|_{V}^{2}\right)\right|_{e^{i t a} \cdot x}
\end{aligned}
$$

where $\|\cdot\|_{V}$ is the Kähler metric on $V$.

If $\tilde{a}_{x}=0$ then $L_{a}(t)$ will be constant and not proper, hence $x$ cannot have any infinitesimal stablizers and $q_{a}(t)$ is strictly convex. The function $e^{L_{a}}$ is also strictly convex. This implies that the Hessian of $S_{x}$ at the origin, Hess $\left(S_{x}\right)(0)>$ 0 . But Hess $\left(S_{x}\right)(a)=\operatorname{Hess}\left(S_{e^{i t a} x}\right)(0)$, which by the same analysis must also be positive definite. $S_{x}$ is a strictly convex, proper function so it has exactly one critical point which is an absolute minimum. By equation (1), such a point determines a unique $G$-orbit in the $G^{\mathbb{C}}$-orbit of $x$ on which $\mu \equiv 0$. Conversely, assume $x$ has no infinitesimal stablizers and $G^{\mathbb{C}} \cdot x \cap \mu^{-1}(0)$ is a single $G$-orbit. We need to show that $S_{x}$ is proper, which by above analysis is clear since $S_{x}$ has a unique critical point and is convex along geodesics. Hence $S_{x}$ is proper.

Now let us bring the previous setup into our situation. Here our projective manifold $V$ is Grass $(r, N)$ the complex Grassmanian with the standard FubiniStudy Kähler form. $G:=S U(N)$ and $G^{\mathbb{C}}:=S L(N)$, in this case the moment map is given by the following formula:

$$
\mu([A]):=A_{N \times r} A_{r \times N}^{*}-\frac{r}{N} I_{N \times N} \in \sqrt{-1} s u(N),
$$

where $A_{N \times r}:=\left[\overrightarrow{a_{1}}, \ldots, \overrightarrow{a_{r}}\right]$, and $\left\{\overrightarrow{a_{1}}, \ldots, \overrightarrow{a_{r}}\right\} \in \mathbb{C}^{N}$ is an orthonormal basis for the $r$-plane in Grass $(r, N)$, in particular $A_{r \times N}^{*} A_{N \times r}=I_{r \times r}$. We know that (Grass $(r, N), L)$ is quantized, where the line bundle $L$ is exactly the determinant line bundle of the universal quotient bundle equipped with the Fubini-Study metric $h_{F S}$ induced from the standard metric on $\mathbb{C}^{K+1}$.

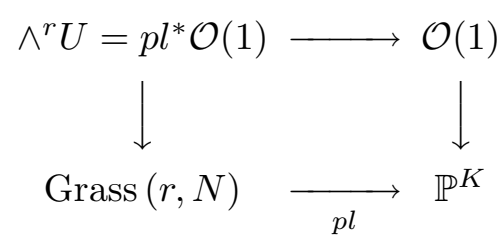

where $K=\left(\begin{array}{c}N \\ r\end{array}\right)-1$ and $p l$ is the Plücker embedding. We want to write down the distortion function $S_{x}$ for the action of $S L(N)$ extending $S U(N)$.

Suppose that $x:=\left\{\overrightarrow{a_{1}}, \ldots, \overrightarrow{a_{r}}\right\} \in \operatorname{Grass}(r, N)$, Then

$$
S_{x}(g):=\frac{h_{F S}\left(g \cdot \xi_{x}\right)}{h_{F S}\left(\xi_{x}\right)}:=\frac{\left|\overrightarrow{g \cdot \overrightarrow{a_{1}} \wedge \ldots \wedge g \cdot \overrightarrow{a_{r}}}\right|_{\mathbb{C}^{K+1}}}{\left|\overrightarrow{a_{1} \wedge \ldots \wedge \overrightarrow{a_{r}}}\right|_{\mathbb{C}^{K+1}}}
$$


where $\xi_{x}$ is a lift of $x$ in $L$, and $|\cdot|_{\mathbb{C}^{K+1}}$ is the standard euclidean metric and $\bar{x}$ means the lift of $x \in \mathbb{P}^{K}$ in $\mathbb{C}^{K+1}$. Clearly that $S_{x}(g)$ is independent of the choice of the lift. Now we are trying to relate this setting to our bundle case. Let us consider the infinite-dimensional manifold $\operatorname{Map}(X, \operatorname{Grass}(r, N))$ of smooth maps from $X$ to the Grassmannian. The tangent space at the point $f \in \operatorname{Map}(X, \operatorname{Grass}(r, N))$ is given by $v \in \Gamma\left(X, f^{*} T \operatorname{Grass}(r, N)\right)$. We define the symplectic form as follows to make it a symplectic manifold:

$$
\Omega(v, w)=\int_{X} \omega_{F S}(v, w) d \operatorname{Vol}_{\mathrm{X}}
$$

where $v, w \in \Gamma\left(X, f^{*} T \operatorname{Grass}(r, N)\right)$ and $\omega_{F S}$ is the Fubini-Study Kähler form on $\operatorname{Grass}(r, N)$.

Lemma 3.3. $\Omega$ is closed.

Proof. We have the following diagram:

$$
\begin{gathered}
\operatorname{Map}(X, \operatorname{Grass}(r, N)) \times X \stackrel{e v}{\longrightarrow} \operatorname{Grass}(r, N) \\
\downarrow^{\pi} \\
\operatorname{Map}(X, \operatorname{Grass}(r, N))
\end{gathered}
$$

where $e v(f, x)=f(x), \pi(f, x)=f$; so

$$
\Omega=\pi_{*}\left(e v^{*} \omega_{F S} \wedge d \mathrm{Vol}_{X}\right)
$$

clearly it is closed.

Now $S U(N)$ action on $\operatorname{Map}(X, \operatorname{Grass}(r, N))$ is induced by the action on Grass $(r, N)$; it is a Hamiltonian action and the moment map is given by the following:

\section{Lemma 3.4.}

$$
\mu_{\text {Map }(X, \operatorname{Grass}(r, N))}=\int_{X} \mu_{\operatorname{Grass}(r, N)} d \operatorname{Vol}_{X}
$$

Proof. At the point $f \in \operatorname{Map}(X, \operatorname{Grass}(r, N))$ we have:

$$
\begin{aligned}
i_{\tilde{a}} \Omega & =\int_{X} i_{\tilde{a}} \omega_{F S} d \operatorname{Vol}_{X} \\
& =\int_{X} f^{*}\left(d\left\langle\mu_{\operatorname{Grass}(r, N)}, a\right\rangle\right) \wedge d \operatorname{Vol}_{X} \\
& =\pi_{*}\left(e v^{*}\left(d\left\langle\mu_{\operatorname{Grass}(r, N)}, a\right\rangle\right) \wedge d \operatorname{Vol}_{X}\right) \\
& =d\left\langle\pi_{*}\left(e v^{*} \mu_{\operatorname{Grass}(r, N)} d \operatorname{Vol}_{X}\right), a\right\rangle
\end{aligned}
$$

where $a \in \operatorname{sl}(N), \tilde{a} \in T$ Grass and $\langle\cdot, \cdot\rangle$ is the pairing between $\mathfrak{g}$ and $\mathfrak{g}^{*}$. Ad Ad $^{*}$ equivariance is clear.

Remark 3.3. Actually it is easy to see that $\operatorname{Map}(X, \operatorname{Grass}(r, N))$ is a infinite dimensional Kähler manifold (c.f. $[\mathrm{H}])$. 
Let $\operatorname{Map}^{E}$ denote space of holomophic maps from $X$ to $\operatorname{Grass}(r, N)$ with the homotopy type governed by bundle $E$, which is a finite dimensional space. At smooth points it is actually a Kähler submanifold of $\operatorname{Map}(X, \operatorname{Grass}(r, N))$. Following the notation in Definition 2.2, for $k>>1$ we can easily get the following

Remark 3.4. There is a canonical injection $j$ such that the following diagram commutes.

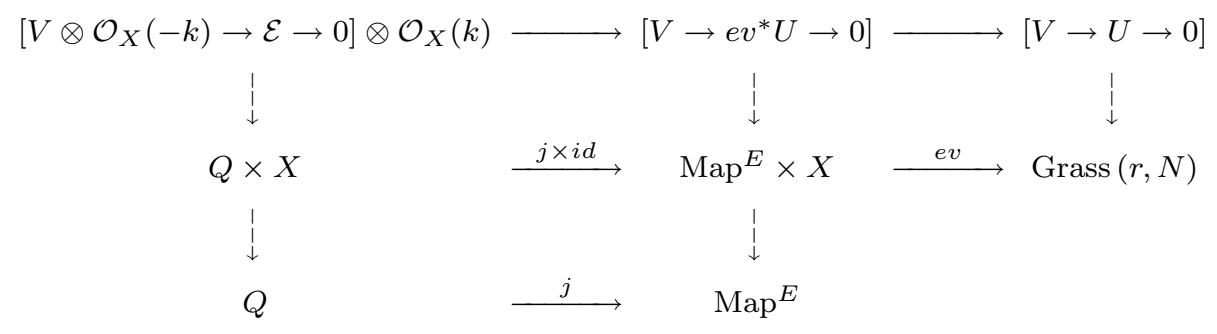

Where $Q \subset$ Quot $(\mathcal{H}, p)$ is the open subset corresponding to the irreducible holomorphic vector bundles over $X$.

Let us introduce the functional we are going to use

$$
L(g):=\int_{X} \log S_{x}(g) d \operatorname{Vol}_{X}
$$

So $L: S L(N) / S U(N) \longrightarrow \mathbb{R}$, and critical points of L are exactly the zeroes of the moment map $\mu_{\operatorname{Map}(X, \operatorname{Grass}(r, N))}$.

\section{Lemma 3.5.}

$$
\frac{d^{2} L}{d t^{2}}=\int_{X}\|\tilde{a}\|_{\text {Grass }}^{2} d \operatorname{Vol}_{X}
$$

along the geodesic which is the translate of $\exp ($ ita), for $a \in \mathfrak{g}$, where $\tilde{a}$ is the induced vector field on $\operatorname{Grass}(r, N)$, and $\|\cdot\|_{\text {Grass }}$ is the standard metric on $\operatorname{Grass}(r, N)$. In particular $L(g)$ is strictly convex along geodesics of $S L(N) / S U(N)$.

Proof. It follows directly from Lemma 3.1 and 3.2.

Now bring the vector bundle into our picture. Suppose $E$ is a vector bundle over $X$, and suppose $k>M$ where $M$ is the constant in Theorem 2.3. Then we can use global sections of $E(k)$ to embed $X$ into $\operatorname{Grass}(r, N)$ where $N=p(k)$ and $p(k)$ is the Hilbert polynomial of $E$. So we get the following diagram:

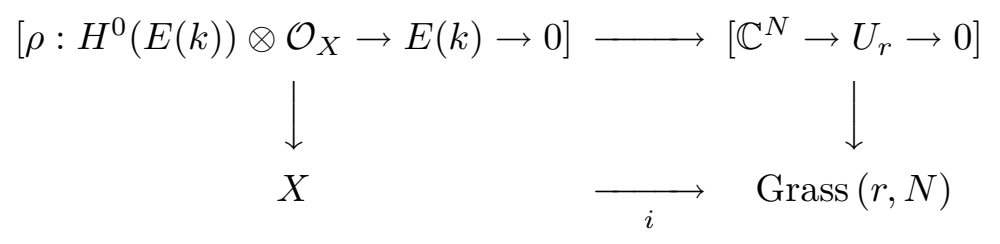


Remark 3.5. Fix a Hermitian metric on $E$ and $\mathcal{O}_{X}(1)$. This induces a Hermitian metric on $E(k)$, and by intergrating over $X$ we get an $L^{2}$-metric on $H^{0}(E(k))$. Pick an orthonormal basis $\left\{Z_{1}, \ldots, Z_{N}\right\}$ with respect to this $L^{2}$ metric, this gives an embedding of $X$ into $\operatorname{Grass}(r, N)$. Under this embedding, $\left\{Z_{1}, \ldots, Z_{N}\right\}$ can be viewed as the restriction of the coordinate sections of the universal quotient bundle $U_{r} \rightarrow \operatorname{Grass}(r, N)$ and this embedding can be written down explicitly as long as we have fixed a local basis of sections. Let $\left\{e_{1}, \ldots, e_{r}\right\}$ be a local orthonormal frame of $E(k)$ in a neighbourhood of a point. We have the following matrix representative.

$$
\left(\begin{array}{c}
Z_{1} \\
Z_{2} \\
\vdots \\
Z_{N}
\end{array}\right)=\left(\begin{array}{ccc}
a_{11} & \ldots & a_{1 r} \\
\ldots \ldots \ldots \ldots & \ldots \\
a_{N 1} & \ldots & a_{N r}
\end{array}\right)\left(\begin{array}{c}
e_{1} \\
e_{2} \\
\vdots \\
e_{r}
\end{array}\right)
$$

Set

$$
A(x):=\left(\begin{array}{lll}
a_{11}(x) & \ldots & a_{1 r}(x) \\
\ldots \ldots & \ldots \ldots & \ldots \ldots \\
a_{N 1}(x) & \ldots & a_{N r}(x)
\end{array}\right)_{N \times r}
$$

Actually we could use $A_{0}:=A_{N \times r}\left(A^{*} A\right)_{r \times r}^{-\frac{1}{2}}\left(\right.$ because $\left.A_{0}^{*} A_{0}=I\right)$. It is clear that $[A]=\left[A_{0}\right] \in \operatorname{Grass}(r, N)$ is independent of the choice of the orthonormal frame we choose. We can write down the Fubini-Study inner product $\langle\cdot, \cdot\rangle_{F S}$ on $U_{r} \rightarrow \operatorname{Grass}(r, N)$ of sections $\left\{Z_{1}, \ldots, Z_{N}\right\}$ at the point $[A] \in \operatorname{Grass}(r, N)$ :

$$
\left.\left(\begin{array}{ccc}
\left\langle Z_{1}, Z_{1}\right\rangle_{F S} & \ldots & \left\langle Z_{1}, Z_{N}\right\rangle_{F S} \\
\ldots \ldots \ldots \ldots \ldots & \ldots & \ldots \ldots \ldots \\
\left\langle Z_{N}, Z_{1}\right\rangle_{F S} & \ldots & \left\langle Z_{N}, Z_{N}\right\rangle_{F S}
\end{array}\right)\right|_{x \in X}=\left.A\left(A^{*} A\right)^{-1} A^{*}\right|_{x \in X}
$$

where $A^{*}:=\bar{A}^{t}$. The above formula gives the relation between the FubiniStudy metric and the original metric on E. Note that $A(x) A(x)^{*}$ is independent of the choice of the orthonormal frame and it is the inner product matrix $\left\{\left\langle Z_{i}(x), Z_{j}(x)\right\rangle_{h}\right\}$ with respect to the original Hermitian metric $h$ on $E(k)$. Also we see that $A(x)^{*} A(x)$ is well-defined as a bundle morphism (because a change of the frame will end up with a conjugation on this matrix which obeys the transformation law). Now if we have $A(x)^{*} A(x) \equiv$ Const. I, we see from the above formula that the original metric of $E(k)$ is actually a scalar multiple of the Fubini-Study metric which is induced from the embedding $X \rightarrow \operatorname{Grass}(r, N)$ via the sections $Z_{i}^{\prime} s$, and Fubini-Study metric is induced from a Hermitian inner product on the space $H^{0}(E(k))$ where $\left\{Z_{1}, \ldots, Z_{N}\right\}$ is an orthonormal basis.

$S L(N)$ acts on this embedding; the action on the function $L(g)$ can be expressed in the following way: 


$$
\begin{aligned}
L(g): & =\int_{X} \log \frac{\sum_{\left(i_{1}, i_{2}, \ldots, i_{r}\right) \in\{1,2, \ldots, N\}}\left|g \cdot Z_{i_{1}}(x) \wedge \cdots \wedge g \cdot Z_{i_{r}}(x)\right|^{2}}{\sum_{\left(i_{1}, i_{2}, \ldots, i_{r}\right) \in\{1,2, \ldots, N\}}\left|Z_{i_{1}}(x) \wedge \cdots \wedge Z_{i_{r}}(x)\right|^{2}} d \operatorname{Vol}_{X} \\
& =\int_{X} \log \sum_{\left(i_{1}, i_{2}, \ldots, i_{r}\right) \in\{1,2, \ldots, N\}}\left\|g \cdot Z_{i_{1}}(x) \wedge \cdots \wedge g \cdot Z_{i_{r}}(x)\right\|_{F S}^{2} d \operatorname{Vol}_{X}
\end{aligned}
$$

Here we abuse slightly the notation, where $|\cdot|$ is the standard euclidean norm in $\mathbb{C}^{K+1}$, and the $Z_{i}^{\prime} s$ are as above, $Z_{i_{1}} \wedge \ldots \wedge Z_{i_{r}}$ is the lift of the corresponding point in $\mathbb{P}^{K}$ and $g \in S L(N)$. What we really have in mind in fact is the following diagram:

$$
\begin{aligned}
& X \stackrel{i}{\longrightarrow} \operatorname{Grass}(r, N) \stackrel{p}{\longrightarrow} \mathbb{P}^{K} \\
& x \stackrel{p \circ i}{\longrightarrow}\left[Z_{1}(x) \wedge \ldots \wedge Z_{r}(x), \ldots, Z_{i_{1}}(x) \wedge \ldots \wedge Z_{i_{r}}(x), \ldots\right] \in \mathbb{P}^{K}
\end{aligned}
$$

Theorem 3.1. Suppose $E$ is a vector bundle over $X$, then $E$ is Gieseker stable if and only if there is a number $M$ such that for $k>M$, the function $L: S L(N) / S U(N) \longrightarrow \mathbb{R}$ corresponding to the embedding with the fixed basis $\left\{Z_{1}, \ldots, Z_{N}\right\} \in H^{0}(E(k))$ has a unique critical point $\left[g_{0}\right] \in S L(N) / S U(N)$, where $g_{0} \in S L(N)$.

Before we prove the theorem, let us use Kempf-Ness's criterion in Section 2 and Lemma 3.1 to reformulate Theorem 2.3. Recall that the Hermitian metric $h$ on $E$ induces an $L^{2}$-metric on $H^{0}(E(k))$. We pick an orthonormal basis of $H^{0}(E(k))$ with respect to this $L^{2}$-metric to give an embedding $i: X \longrightarrow$ Grass $(r, N)$, so $E(k) \cong i^{*}\left(U_{r}\right)$ where $U_{r}$ is the universal quotient bundle over Grass $(r, N)$, it has the induced Fubini-Study metric from the Grass $(r, N)$ which is of course different from the original one in general. Integrating the FubiniStudy metric on $\operatorname{det} E(k) \cong i^{*}\left(\operatorname{det} U_{r}\right)$ over $X$ gives an $L^{2}$-metric on $H^{0}(\operatorname{det} E(k))$. So we get a metric $\|\cdot\|_{V}$ on $V:=\operatorname{Hom}\left(\wedge^{r} H^{0}(E(k)), H^{0}(\operatorname{det}(E(k)))\right), T(E(k)) \in$ $V, G^{\mathbb{C}}=S L(N)$. Theorem 2.3 can be formulated as the following:

Proposition 3.2. There is an $M$ so that if $k>M$, then $E(k)$ is Gieseker stable if and only if :

$$
\begin{gathered}
\|g \cdot T(E(k))\|_{V}^{2}:= \\
\int_{X} \sum_{\left(i_{1}, i_{2}, \ldots, i_{r}\right) \in\{1,2, \ldots, N\}}\left\|g \cdot Z_{i_{1}}(x) \wedge g \cdot Z_{i_{2}}(x) \wedge \ldots \wedge g \cdot Z_{i_{r}}(x)\right\|_{F S}^{2} d \mathrm{Vol}_{X}
\end{gathered}
$$

is a proper function on $S L(N) / S U(N)$.

Proof. Let $\left(W_{i}^{r_{i}}, h_{i}\right), i=1,2$ be two finite dimensional vector spaces of dimension $r_{i}$ with Hermitian metrics $h_{i}$ and $T: W_{1} \rightarrow W_{2}$ be a linear map. Then the operator norm $\|T\|_{h_{1}, h_{2}}$ which depends on $h_{1}$ and $h_{2}$ can be computed as follows. 
Pick an orthonormal basis of $\left\{w_{1}, \cdots, w_{r_{1}}\right\} \in W_{1}^{r_{1}}$, then

$$
\|T\|_{h_{1}, h_{2}}^{2}:=\sum_{i=1}^{r_{1}}\left\|T\left(w_{i}\right)\right\|_{h_{2}}^{2}
$$

In our case we use the Hermitian metric on $E$ which induces a Hermitian metric on $H^{0}(E(k))$, and hence on $\wedge^{r} H^{0}(E(k))$. For the metric on $\operatorname{det}(E(k))$ we use the Fubini-Study metric induced from the embedding into $\mathbb{P}^{K}$ instead of the induced metric from $h$ on $E$. The Fubini-Study metric also induces an $L^{2}$-metric on $H^{0}(\operatorname{det}(E(k))$, so we get a Hermitian metric on

$$
V:=\operatorname{Hom}\left(\wedge^{r} H^{0}(E(k)), H^{0}(\operatorname{det}(E(k)))\right)
$$

Using Lemma 3.1 completes the proof.

Lemma 3.6. Let $(L, h)$ be a holomorphic line bundle over $X$ with a Hermitian metric $h$. Let $s \in H^{0}(L)$ satisfy the following condition:

$$
\int_{X}\|s\|_{h}^{2} d \operatorname{Vol}_{X} \geq 1
$$

Then there is a universal constant $C>-\infty$ depending only on $L$, in particular independent of the choice of $s$, such that:

$$
\int_{X} \log \|s\|_{h}^{2} d \operatorname{Vol}_{X}>C
$$

Proof. Clearly, we only need to show the case where

$$
\int_{X}\|s\|_{h}^{2} d \mathrm{Vol}_{X}=1
$$

since $\log a \geq 0$ for $a \geq 1$, that boils down to showing that the function

$$
\begin{gathered}
F(s):=\int_{X} \log \|s\|_{h}^{2} d \operatorname{Vol}_{X} \\
F: H^{0}(L)-\{0\} \rightarrow \mathbb{R}
\end{gathered}
$$

is continuous. Suppose

$$
\lim _{i \rightarrow \infty} \int_{X}\left\|s_{i}-s\right\|_{h}^{2} d \mathrm{Vol}_{X}=0
$$

Since $H^{0}(L)$ is a finite dimensional vector space, all norms are equivalent. In particular

$$
\lim _{i \rightarrow \infty}\left\|s_{i}-s\right\|_{C^{2}}=0
$$


Now let $D:=\left\{s^{-1}(0) \subset X\right\}$, and $B_{\delta}(D)$ be a tubular neighborhood of $D$ of radius $\delta$, then we have

$$
\begin{aligned}
F\left(s_{i}\right)-F(s) & =\int_{X-B_{\delta}(D)} \log \frac{\left\|s_{i}\right\|_{h}^{2}}{\|s\|_{h}^{2}} d \operatorname{Vol}_{X} \\
& +\int_{B_{\delta}(D)} \log \left(\left\|s_{i}\right\|_{h}^{2}\right) d \operatorname{Vol}_{X} \\
& -\int_{B_{\delta}(D)} \log \left(\|s\|_{h}^{2}\right) d \operatorname{Vol}_{X}
\end{aligned}
$$

For any given $\epsilon>0$ we first pick a $\delta>0$ such that the third term is less than $\epsilon / 8$, which is possible because $\log \|s(x)\|_{h} \in L^{1}(X)$ for $s \in H^{0}(L)$. Fix this $\delta>0$ we can choose $M>0$ such that for $i>M$ we have the second term less than $\epsilon / 4$; we can do this because of (17). Also use (17) we can make the first term small enough say $<\epsilon / 2$. The first term goes to 0 as $i \rightarrow \infty$. So the sum of these three terms will be less than $\epsilon$. This completes the proof of continuity of the function $F$. Now since holomorphic sections satisfying the equation (13) with equality lie on the unit sphere of a finite dimensional vector space which is compact, so clearly $F$ has a lower bound which only depends on $(L, h)$ and the geometry of X.

Remark 3.6. Notice the above proof does not depend on which volume form we choose. Actually, we only need a semipositive volume form which is strictly postive at one point.

Proof of Theorem 3.1. In order to show that $L$ has a critical point, since $L$ is convex along geodesics on the symmetric space $S L(N) / S U(N)$, the only thing we need to show is that $L$ is bounded from below and proper if $E(k)$ is Gieseker stable. We need to show that for any sequence $G^{\mathbb{C}} \ni g_{i} \rightarrow \infty, L\left(g_{i}\right) \rightarrow+\infty$. Let:

$p(g):=\int_{X} \sum_{\left(i_{1}, i_{2}, \ldots, i_{r}\right) \in\{1,2, \ldots, N\}}\left\|g \cdot Z_{i_{1}}(x) \wedge g \cdot Z_{i_{2}}(x) \wedge \ldots \wedge g \cdot Z_{i_{r}}(x)\right\|_{F S}^{2} d \operatorname{Vol}_{X}$

so there must be a $\sqrt{\frac{K+1}{p(g)}}\left(g \cdot Z_{i_{1}} \wedge \ldots \wedge g \cdot Z_{i_{r}}\right) \in H^{0}(\operatorname{det} E(k))$ satisfying (13)

of Lemma 3.6, where $K=\left(\begin{array}{c}N \\ r\end{array}\right)-1$ as before. So we have the following:

$$
L(g)>\operatorname{Vol}(X) \log p(g)+C
$$

where $C$ is the constant in Lemma 3.6 . Now since $p(g)$ is proper and convex on $S L(N) / S U(N)$ by Proposition 3.2 and Theorem 2.3, so $p(g) \rightarrow+\infty$ as $g \rightarrow \infty$, which implies $L(g)$ is a proper function on $S L(N) / S U(N)$. $L(g)$ is strictly convex by Lemma 3.5 , so it has a unique critical point $g_{0}$. 
Conversely, if $L$ has a critical point, then it is proper since it is strictly convex. But if $E(k)$ is not Gieseker stable then $T(E)$ is not stable, so by the analytic Hilbert-Mumford criterion there must be a one parameter subgroup $\lambda(t): t \rightarrow$ $e^{i a t} \subset S L(N), a \in \operatorname{sl}(N)$ such that along this $t \in i \mathbb{R}, p(\lambda(t))$ is not proper, which contradicts the assumptions on $L$.

Remark 3.7. Following from Remark 3.6, it is easy to see that actually we can loose our assumption on the regularity of $E$. In other words, the proof of above theorem clearly goes through as well for torsion free sheaves, thanks to Remark 2.2. In that case we have a holomophic map $i: X_{\text {reg }} \rightarrow \operatorname{Grass}(r, N)$, where $X_{\text {reg }}$ is an open dense set in $X$ over which $E$ is locally free, the closure of image of $X_{\text {reg }}$ under $i$ (which is not necessarily smooth) can still be moved to a balance position under the $S L(N)$ action if $E$ is stable.

Now let us give two examples to illustrate conceptually how the proof of the theorem goes.

Example 3.1. Let us consider the line bundle case, and $k>>1$. Since line bundles are always stable, we should get that $L$ is always proper and bounded from below. Let $N=\operatorname{dim} H^{0}(E(k))$ and $\left\{Z_{1}, \cdots, Z_{N}\right\}$ be a basis of $H^{0}(E(k))$. Choose a one-parameter subgroup

$$
\lambda: \mathbb{G}_{m} \ni t \rightarrow \operatorname{diag}\left[t^{w_{1}}, \cdots, t^{w_{N}}\right] \in S L(N)
$$

where $w_{i}^{\prime} s$ are the weights. The restriction of $L$ to $\lambda\left(\mathbb{G}_{m}\right)$ is:

$$
L(t)=: \int_{X} \log \left(t^{2 w_{1}}\left\|Z_{1}(x)\right\|_{F S}^{2}+\cdots+t^{2 w_{N}}\left\|Z_{N}(x)\right\|_{F S}^{2}\right) d \operatorname{Vol}_{X}
$$

which is proper and bounded from below on $\mathbb{G}_{m}$, because

$$
\begin{aligned}
& t^{2 w_{1}}\left\|Z_{1}(x)\right\|_{F S}^{2}+\cdots+t^{2 w_{N}}\left\|Z_{N}(x)\right\|_{F S}^{2} \\
\geq & N\left(t^{2 w_{1}}\left\|Z_{1}(x)\right\|_{F S}^{2} \cdots t^{2 w_{N}}\left\|Z_{N}(x)\right\|_{F S}^{2}\right)^{1 / N} \\
= & N\left(\left\|Z_{1}(x)\right\|_{F S}^{2} \cdots\left\|Z_{N}(x)\right\|_{F S}^{2}\right)^{1 / N}
\end{aligned}
$$

since $w_{1}+\cdots+w_{N}=0$.

Example 3.2. Now let us see why the above situation will not be the case for higher rank bundles. Let us see for instance $r k(E(k))=2$ and the notations are as above. The restriction of $L$ to $\lambda\left(\mathbb{G}_{m}\right)$ becomes:

$$
L(t)=: \int_{X} \log \left(\sum_{i<j} t^{2\left(w_{i}+w_{j}\right)}\left\|Z_{i} \wedge Z_{j}\right\|_{F S}^{2}\right) d \operatorname{Vol}_{X}
$$

Although $\sum_{i<j}\left(w_{i}+w_{j}\right)=0$, it is possible that some of $Z_{i}(x) \wedge Z_{j}(x) \equiv 0$ on $X$. So we might have all the remaining weights $\left(w_{i}+w_{j}\right)$ except those $(i, j)$ such that $Z_{i}(x) \wedge Z_{j}(x) \equiv 0$ on $X$ have the same sign, that will make $L(t)$ have no lower bound. Fortunately, these bad situations are exactly avoided by the stability condition given by Gieseker (Theorem 2.3). Geometrically that means not too many sections concentrate in a strictly lower rank bundle, in this case a 
line bundle. That is exactly the condition $Z_{i}(x) \wedge Z_{j}(x) \equiv 0$, since that means these two sections are co-linear. This also reflects that the slope criterion means avoiding too many sections lying in a strictly lower rank bundle.

Definition 3.5. Suppose $E$ is a vector bundle over $X$, then $E$ is called balanced if there is a number $M$ such that for $k>M$, we can find a $g \in S L(N)$, where $N:=\operatorname{dim} H^{0}(X, E(k))$ such that

$$
\int_{g \cdot X} \mu_{\mathrm{Grass}(r, N)} d \mathrm{Vol}_{X}=0
$$

where $g \cdot X$ means the image of $X \subset \operatorname{Grass}(r, N)$ under the action of $g$, and $g$ is called the balanced point of this embedding $X \rightarrow \operatorname{Grass}(r, N)$.

Proof of Theorem 1.1. From the proof of Lemmas 3.1 and 3.2, we have

$$
\frac{d L}{d g}:=\int_{g \cdot X} \mu_{\operatorname{Grass}(r, N)} d \operatorname{Vol}_{X}
$$

so the unique critical point of $L(g)$ exactly corresponds to the balance point.

Now we can conclude this section by the following corollary.

\section{Corollary 3.1.}

$$
\mathcal{M}^{0}(X, p(k)) \cong \mu_{j(Q)}^{-1}(0) / / S U(N)
$$

where $\mathcal{M}^{0}(X, p(k))$ is the open subset of $\mathcal{M}(X, p(k))$ coresponding to irreducible locally free sheaves, $\mu_{j(Q)}$ is the restiction of $\mu_{\mathrm{Map}_{(X, \mathrm{Grass}(r, N))}}$ to the image of $Q$ under the map $j$ (c.f. Remark 3.4).

Remark 3.8. This result is the vector bundle analogue of the interesting result proved by Luo (c.f. [Luo]), where he characterized the Gieseker-Mumford stability for a polarized projective manifold by a similar balanced condition. But there is a key difference between the manifold case and the bundle case: in the manifold case the volume form is inherited from $\mathbb{C P}^{N-1}$, thus changing under the $S L(N)$ action; in the bundle case, the volume form is fixed. This is why the stability issue for manifolds is, in some sense, more nonlinear than that for bundles. Also his approach is completely different from ours.

\section{Differential Geometric Picture}

In this section we are going to give the differential geometric interpretation of the results in previous sections. Let $(E, h)$ be a holomorphic vector bundle over $X$ with a Hermitian metric $h$.

Definition 4.1. Let $L^{2}(X, E)$ denote the $L^{2}$ sections of the vector bundle $E$ and let $T$ be the $L^{2}$-orthogonal projection with respect to $h$.

$$
T: L^{2}(X, E) \rightarrow H^{0}(X, E)
$$


Let $\Pi(x, y)$ denote the Bergman kernel of the Toeplitz operator $T$

$$
T s(x):=\int_{X} \Pi(x, y) s(y) d y
$$

For any $L^{2}$-orthonormal basis $\left\{s_{1}, \cdots, s_{N}\right\}$ of $H^{0}(X, E)$ we have

$$
B(x):=\Pi(x, x)=\sum_{i=1}^{N}\left\langle s_{i}(x), \cdot\right\rangle_{h} s_{i}(x): E_{x} \rightarrow E_{x}
$$

Now we can reformulate the main theorem as the following:

Proposition 4.1. Suppose $E$ is a holomorphic vector bundle over $X$, then $E$ is Gieseker polystable if and only if there is a number $M$ such that for $k>M$, we can find a metric on $E(k)$ such that $B=$ Constant $\cdot I_{E(k)}$, where $I_{E(k)}$ is the identity map.

Proof. Note that from Theorem 1.1 we see that $E(k)$ is Gieseker stable if and only if the integral of the moment map $\mu_{\text {Grass }}$ over $g_{0} \cdot X \subset \operatorname{Grass}(r, N)$ is 0 , which means $\left\{Z_{1}, \cdots, Z_{N}\right\} \subset H^{0}(X, E(k))$ (up to a scalar) is an $L^{2}$-orthonormal basis with respect to the Fubini-Study metric on $E$ over $g_{0} \cdot X$, and

$$
B(x)=\Sigma_{i=1}^{N}\left\langle Z_{i}(x), \cdot\right\rangle_{F S} Z_{i}(x) \equiv \text { Const. } \cdot I_{E}
$$

because $Z_{i}^{\prime} s$ are coordinate sections on $U_{r} \rightarrow \operatorname{Grass}(r, N)$.

Conversely, if $B(x) \equiv$ Const $\cdot I_{E}$ then from Remark 3.5 we know that the Fubini-Study metric induced from the embedding $X \rightarrow \operatorname{Grass}(r, N)$ is a scalar multiple of the original Hermitian metric $h$ on $E$, and that means $\left\{Z_{1}, \cdots, Z_{N}\right\} \subset$ $H^{0}(X, E(k))$ by our choice (up to a scalar) is an orthonormal basis with respect to the Fubini-Study metric which is equivalent to :

$$
\frac{d L}{d g}:=\int_{g \cdot X} \mu_{\operatorname{Grass}(r, N)} d \operatorname{Vol}_{X}=0
$$

so $E$ is Gieseker stable by Theorem 1.1.

Next we are going to explain the relation among Bergman kernel, heat kernel and the equation derived by Leung. Let us recall the heat kernel of the operator $\bar{\partial}$ on $\Omega^{0, *}(X, E)$ and the local Atiyah-Singer index theorem. The Dirac operator for Kähler manifolds can be identified as $D=\sqrt{2}\left(\bar{\partial}+\bar{\partial}^{*}\right)$ acting on $\Omega^{0, *}(X, E)$. Let $H_{t}(x, x)$ denote the restriction of the heat kernel of $D^{2}$ to the diagonal of $X \times X$. Then by the local Atiyah-Singer index theorem (c.f.[BGV]), the limit of the supertrace of $H_{t}(x, x)$ exists as $t \rightarrow 0$. More precisely:

$$
\lim _{t \rightarrow 0^{+}} \operatorname{Tr}_{s} H_{t}(x, x)=\left[e^{R(E)} T d_{X}\right]^{(n, n)}
$$

where $R(E)$ is the curvature form of $E$.

Let $L:=\mathcal{O}_{X}(1)$ and fix a Hermitian metric $h$ on $L$ such that the Kähler form $\omega$ on $X$ is given by $\operatorname{Ric}(h)$. In [Le] Leung proved the following theorem. 
Theorem 4.1. Let $E$ be an irreducible sufficiently smooth holomorphic vector bundle over a polarized projective manifold $\left(X, \mathcal{O}_{X}(1)\right)$. Then $E$ is Gieseker stable if and only if there exists a Hermitian metric on $E$ which solves the following almost Hermitian-Einstein equation:

$$
\left[e^{R(E)+k \omega \cdot I_{E}} T d_{X}\right]^{(n, n)}=\frac{1}{r k(E)} \chi(X, E(k)) \frac{\omega^{n}}{n !} I_{E} .
$$

Let $H_{t}^{k}$ denote the heat kernel for bundle $E(k)$, then we have

$$
\lim _{t \rightarrow \infty} \operatorname{Tr}_{s} H_{t}^{k}(x, x)=\lim _{t \rightarrow \infty} H_{t}^{k}(x, x)=B(x) \frac{\omega^{n}}{n !}
$$

where the first identity follows from the vanishing of the $H^{i}(X, E(k))$ for $i>0$ and $k$ sufficient large.

Note that for fixed $k$, the metric on $E$ which solves Leung's equation is in general different from the metric satisfying $B \equiv$ Const. Nevertheless, as $k \rightarrow \infty$, they should be getting closer and closer in the following sense:

Conjecture 1. We have the following asymptotic expansion of $B$

$$
\left[e^{R(E)+k \omega \cdot I_{E}} T d_{X}\right]^{(n, n)}=B \frac{\omega^{n}}{n !}+O\left(\frac{1}{k}\right)
$$

as long as the metric satisfies $B \equiv$ Const.

Remark 4.1. Notice the last condition $B \equiv$ Const. is necessary otherwise the statement is not true (c.f. $[\mathrm{Lu}]$ ). From Remark 3.7 we know actually we can get a singular metric on torsion free sheaves as Bando and Siu did in [BS] such that $B(x) \equiv$ Const. - I over the open dense subset of $X$ where $E$ is locally free, and we also have that the curvature is integrable if $E$ is torsion free, and square integrable if $E$ is reflexive. Combine with the conjecture above, naturally we expect that there is an extension of Leung's theorem to the sheaf case as well.

Remark 4.2. Note that our symplectic quotient picture is somewhat different from what Leung did in [Le2] in that we are now dealing with a finite dimensional space of holomorphic maps which is "curved" instead of an infinite dimensional space of connections which is affine as in Leung's picture. Also our approach is sort of family version of GIT quotient which corresponds to the family index theorem approach in [Le2], actually Leung's equation is the Euler-Lagrangian equation of the "transgression" of Riemann-Roch density.

\section{Acknowledgements}

The author is very grateful to Dr. Yang Liu who first brought this problem to his attention and had so many valuable discussions with him. The author would also like to thank Conan Leung, Jun Li, Richard Thomas and Chiu-Chu Liu for their valuable suggestions. Finally, the author wants to thank his advisor S.-T. Yau for his encouragement throughout the work. 


\section{References}

[B] J. Bryan, Symplectic geometry and the relative Donaldson invariants of $\overline{\mathbb{C P}}^{2}$, Forum Math. 9 (1997), no. 3, 325-365.

[BGV] N. Berline, E. Getzler and M. Vergne, Heat kernels and Dirac operators, Grundlehren der Mathematischen Wissenschaften, 298. Springer-Verlag, Berlin, 1992.

[BS] S. Bando, Y. T. Siu, Stable sheaves And Einstein-Hermitian metrics, Geometry and analysis on complex manifolds, 39-50, World Sci. Publishing, River Edge, NJ, 1994.

[D1] S. K. Donaldson, A new proof of a theorem of Narasimhan and Seshadri, J. Differential Geom. 18 (1983), no. 2, 269-277.

[D2] - Anti self-dual Yang-Mills connections over complex algebraic surfaces and stable vector bundles, Proc. London Math. Soc. (3) 50 (1985), no. 1, 1-26.

[D3] _ Infinite determinants, stable bundles and curvature, Duke Math. J. 54 (1987), no. 1, 231-247.

[D4] Geometry in Oxford c. 1980-85, Sir Michael Atiyah: a great mathematician of the twentieth century. Asian J. Math. 3 (1999), no. 1, xliii-xlvii.

[DK] S. K. Donaldson and P. B. Kronheimer, The geometry of four-manifolds, Oxford Mathematical Monographs. Oxford Science Publications. The Clarendon Press, Oxford University Press, New York, 1990.

[G] D. Gieseker, On the moduli of vector bundles on an algebraic surface, Ann. of Math. (2) 106 (1977), no. 1, 45-60.

[H] N. Hitchin, The moduli space of complex Lagrangian submanifolds, Sir Michael Atiyah: a great mathematician of the twentieth century. Asian. J. Math. 3 (1999), no. 1, 77-91.

[HL] D. Huybrechts and M. Lehn, The geometry of moduli spaces of sheaves, Aspects of Mathematics, E31. Friedr. Vieweg \& Sohn, Braunschweig, 1997.

[KN] G. Kempf and L. Ness, The length of vectors in representation spaces, Algebraic geometry (Proc. Summer Meeting, Univ. Copenhagen, Copenhagen, 1978), 233-243, Lecture Notes in Math., 732, Springer, Berlin, 1979.

[Le] N.C. Leung, Einstein type metrics and stability on vector bundles, J. Differential Geom. 45 (1997), no. 3, 514-546.

[Le2] - Symplectic structures on gauge theory, Comm. Math. Phys. 193 (1998), no. 1, $47-67$.

[Lu] Z. Lu, On the lower order terms of the asymptotic expansion of Tian-Yau-Zelditch, Amer. J. Math. 122 (2000), no. 2, 235-273.

[Luo] H. Luo, Geometric criterion for Gieseker-Mumford stability of polarized manifolds, J. Differential Geom. 49 (1998), no. 3, 577-599.

[MFK] D. Mumford, J. Fogarty and F. Kirwan, Geometric Invariant Theory, Third edition. Ergebnisse der Mathematik und ihrer Grenzgebiete (2), 34. Springer-Verlag, Berlin, 1994.

[NS] M. S. Narasimhan and C.S. Seshadri, Stable and unitary bundles on a compact Riemann surface, Proc. Nat. Acad. Sci. U.S.A. 52 (1964), 207-211.

[S] C.T. Simpson, Moduli of representations of the fundamental group of a smooth projuective variety. I, Inst. Hautes Études Sci. Publ. Math. No. 79 (1994), 47-129.

[T] G. Tian On a set of polarized Kähler metrics on algebraic manifolds, J. Differential Geom. 32 (1990), no. 1, 99-130.

[UY] K. Uhlenbeck and S.-T. Yau On the existence of Hermitian Yang-Mills connections on stable vector bundles, Commun. Pure Appl. Math. 39 (1986), no. S, suppl., S257-S293.

[Z] S. Zelditch, Szegö kernels and a theorem of Tian, Internat. Math. Res. Notices 1998 no. $6,317-331$.

Department of Mathematics, Brandeis University, Waltham, MA 02454, U.S.A.

E-mail address: iaowei@math.harvard.edu

E-mail address: xiaowei@euclid.math.brandeis.edu 